\section{Response system processes in absolute judgment*}

\author{
LAWRENCE M. WARD $\dagger$ and G. R. LOCKHEAD \\ Duke University, Durham, North Carolina 27708
}

Consistent relationships are found between $\mathrm{Ss}^{\prime}$ absolute judgments of the value of a stimulus and the previous sequence of both stimuli and responses. The form and magnitude of these sequential effects are shown to depend on the presence or absence of feedback and on task difficulty. The pattern of the sequential effects found allows the conclusion that they are due to purely response-system processes. A two-stage model of the judgment process is proposed, and it is argued that observed assimilative effects account for the central tendency effects observed in category judgments.

The name or response given to a stimulus in an absolute-judgment experiment depends in part on the previous sequence of stimuli. Responses tend to be assimilated toward the value of the immediately previous stimulus and are either contrasted from or assimilated toward earlier stimuli in the sequence, depending on the presence or absence of feedback (knowledge of the "correct" response) (Holland \& Lockhead, 1968; Ward \& Lockhead, 1970). The effects of previous responses in the series of judgments were not reported in the above papers and, in general, have not been the subject of extensive investigation. Garner (1953) and McGill (1957) did show a contingency between the immediately previous response and the response to the current stimulus.

Suggested explanations of the above effects have been based on aberrations in the use of the short-term memory of the previous stimulus as a standard for comparative judgment. The purpose of the present paper is to develop a pattern of sequential effects in different experimental conditions that allows the conclusion that they are due to the use of purely response-system processes in mediating response uncertainty. These response-system processes are shown to take the form of "guessing" strategies.

\section{STUDY 1: SEQUENTIAL EFFECTS WITH AND WITHOUT FEEDBACK}

The purpose of this study was to determine if the presence or absence of feedback affected the form of the effects of previous responses on the current response. These are previously reported data analyzed now for the effects of the previous responses, as well as the already reported stimulus effects. The method,

*This research was supported in part by Grant 5-F01-MH40083-02 from the National Institute of Mental Health.

$\dagger$ Requests for reprints should be sent to Lawrence M. Ward, Department of Psychology, Duke University, Durham, North Carolina 27706. detailed in Ward and Lockhead (1970), is only summarized here.

\section{Method}

$\mathrm{S}$. made absolute judgments of 10 randomly presented $1,000-\mathrm{Hz}$ sinusoids that varied in intensity. Adjacent tones were $1 \mathrm{~dB}$ apart and the total range was approximately from 55 to $64 \mathrm{~dB}$ re .0002 dynes $/ \mathrm{cm}^{2}$. Responses consisted of the numbers 1-10, recorded on an electric typewriter. The stimulus duration was $100 \mathrm{msec}$, and there was a fixed $3.5-\mathrm{sec}$ interval between trials. Three Ss each gave 500 responses per day for 4 consecutive days, following a practice day not reported, with feedback given as an illuminated numeral on every trial. An additional three Ss performed in the same way but with no feedback given.

\section{Results}

Figures 1 and 2 show the effects of the per point.) previous stimuli and those of the previous responses on the response to the current stimulus for the feedback and no-feedback experiments, respectively. The sequential effects are essentially the same for all Ss in this and in the following studies, and the data in these and subsequent figures are averaged over Ss for ease of presentation. The data are also collapsed across adjacent pairs of stimuli for ease of analysis and presentation. The figures take the same form when not averaged in this way.

Each point in the figures represents the average error of the responses to all stimuli that occurred on Trial $\mathrm{N}+\mathrm{K}$ when one or the other of a particular pair of stimuli or responses occurred on Trial $N$. $K$ ranges from one to six, which is as far as the analyses were conducted. For example, the top leftmost point in Fig. 1a represents an average error of +.42 category units on Trial $\mathbf{N}+1$ when the stimulus on Trial $\mathbf{N}$ was a 9 or a 10 . Correspondingly, the same point in Fig. $1 \mathrm{~b}$ represents an average error of +.44 category units on Trial $\mathrm{N}+1$ when the response on Trial $\mathrm{N}$ was a 9 or a 10. For stimuli and responses further back in the sequence than one trial, the average error of response was calculated without respect to what stimuli or responses occurred between the relevant previous trial and the current trial. Since the stimuli were random, it would be expected that each stimulus occurred about equally often in the intervening series of trials. Thus, the data reported here do not refer to the effects of particular sequences of stimuli or responses. These could be calculated from

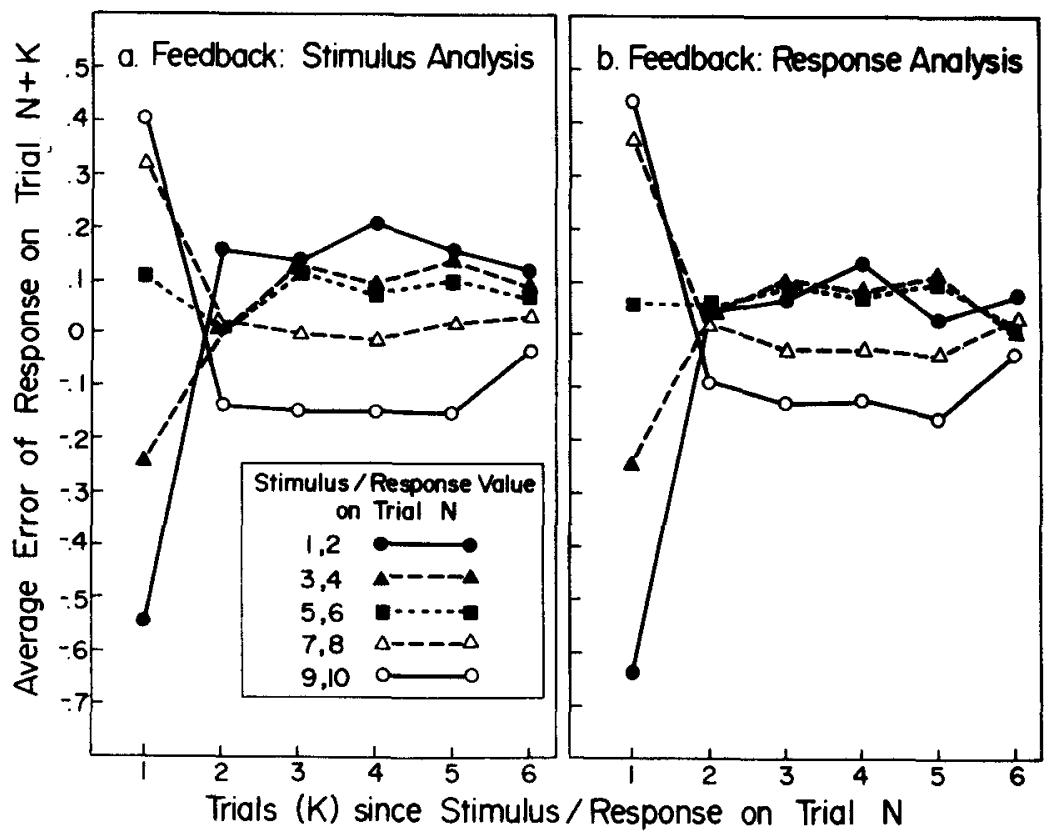

Fig. 1. Average error of the response at $T$ rial $N+K$ attributable to the stimulus/response at Trial $\mathrm{N}$ for the loudness-feedback study. (There are approximately 1,200 observations 


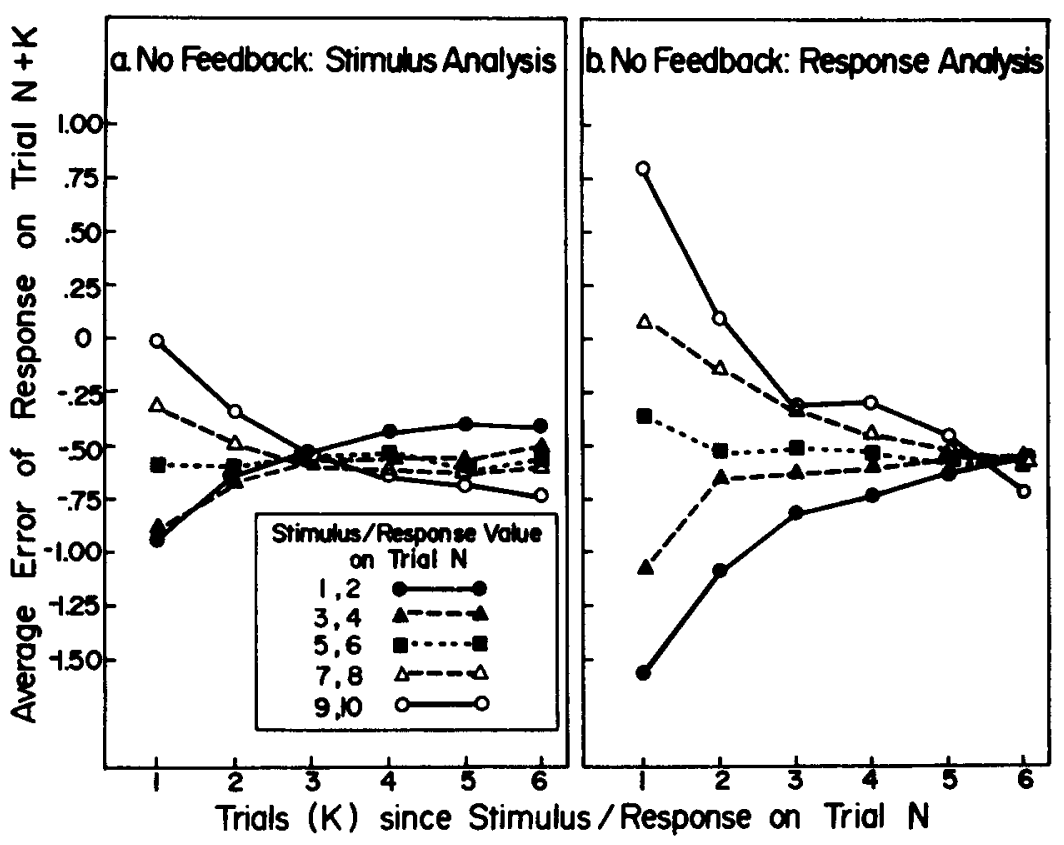

the average results only if there were no interaction between elements and trials.

In the feedback study (Fig. 1), a large valued stimulus or response on Trial $\mathrm{N}$ results in a positive average error of the response on Trial $\mathrm{N}+1$, and small valued stimuli and responses are followed by negative average errors. Thus, responses are "assimilated" toward the values of the previous stimulus and response. But the reverse effect occurs on Trials $\mathrm{N}+2$ through $\mathrm{N}+6$; responses are "contrasted" with stimuli and responses two and more trials back in the sequence. It should be cautioned that the ordering of points in Fig. 1 with respect to the value of the previous stimuli and responses does not imply that, for example, Stimulus 1,2 "pulls" responses toward it more than does Stimulus 5,6. In fact, an approximately equal amount of assimilation is associated with each stimulus (see Fig. 1 in Ward \& Lockhead, 1970). The average effects are ordered as in Fig. 1 because most stimuli that follow 1,2 are larger than 1,2; thus, most of the errors of assimilation are negative, and the average of these is negative. However, half of the stimuli following 5,6 are larger than 5,6, resulting in a negative error, and half are smaller, resulting in a positive average error of the following response. The average of these effects is zero average error.

In the no-feedback study (Fig. 2), responses are assimilated toward stimuli at least two trials back and toward responses at least five trials back in the sequence. Contrast seems to appear for trials further back than mentioned above. The magnitude, defined as the difference in average error between 1,2 and 9,10 , of the assimilative effect of previous responses is considerably greater than that of previous stimuli in this condition.

Information transmission averaged 1.27 and 0.95 bit in the feedback and no-feedback experiments, respectively, and percent correct averaged 36.1 and 27.1. Since stimuli and responses are correlated, there is no way to determine from these data whether the sequential effects are due to stimuli or responses or both.

\section{STUDY 2: SEQUENTIAL EFFECTS IN EASY AND DIFFICULT TASKS}

Performance level affects the correlation between stimuli and responses; as performance approaches chance, the correlation approaches zero. The purpose of this study was to investigate the relation between sequential stimulus effects and sequential response effects when the S's task was the same in two conditions but performance was made different by manipulating the discriminability of the stimuli. A second purpose of the study was to establish the generality of enduring sequential effects beyond loudness judgments. The experiment was conducted for a different purpose and reported earlier, but the data had not been analyzed for the sequential effects reported here. The experimental procedure is summarized here and detailed in Lockhead (1966).

\section{Method}

The stimuli were 10 horizontal black lines .03 in. wide and centered on an $8 \times 8$ in. viewing field. The lines varied in length from 2.0 to $2.9 \mathrm{~cm}$ in $0.1-\mathrm{cm}$ steps and were assigned the response labels 1-10 for the shortest to the longest line. In the easy condition, three Ss each gave 150 responses per day for 8 days and three gave
Fig. 2. Average error of the response at Trial $N+K$ attributable to the stimulus/response at Trial $N$ for the loudness-no-feedback study. (There are approximately 1,200 observations per point.)

150 responses per day for 4 days. The stimuli were presented tachistoscopically for $200 \mathrm{msec}$, and the bluish-white stimulus and adapting fields were easily seen. In the difficult condition, five of the six Ss in the easy condition (the other S had difficulty seeing the stimuli in this condition because of poor acuity and was not used) each gave 150 responses per day for 8 days. In this condition the stimulus duration was reduced to $8 \mathrm{msec}$, and the overall luminance was reduced by means of a 1.0-log unit neutral-density filter. The fields were thus quite dim, and $S$ reported sometimes not seeing the stimulus. Ss partially dark adapted before participating in this condition and were required to respond on all trials. Feedback was given after each response in both conditions.

Both conditions were self-paced, and Ss worked at about the same rate, around $5 \mathrm{sec}$ per stimulus. There was a brief warm-up period before each session. Ss recorded their responses on a keyboard that operated a remote electric typewriter. Thus, Ss could not see the previous sequence of stimuli and responses as they could in Study 1, where they typed their responses and the feedback, when provided, directly on an electric typewriter.

\section{Results}

For the easy condition information transmission averaged over Ss and days was 1.07 bit and percent correct performance was 35.0. In the difficult condition information transmission averaged 0.19 bit and percent correct was 15.0 (chance performance is $10 \%$ ). Thus, the correlation between stimuli and responses is different in the two conditions, while the stimuli were still visible enough to be judged in the difficult condition.

Figures 3 and 4 show the analyses of the data from the easy and difficult conditions, respectively, for the effects of previous stimuli and previous responses on the current response. The form of the sequential effects in the easy condition (Fig. 3) is comparable to that from the loudness-feedback study (Fig. 1), although the magnitude of the assimilation effects is less and the variability of the curves is greater for the line-length study. These differences may be due to the smaller number of trials per day run in this study (150 vs 500 ) or to the longer intertrial time $(5 \mathrm{sec}$ vs $3.5 \mathrm{sec})$. The major effect, 


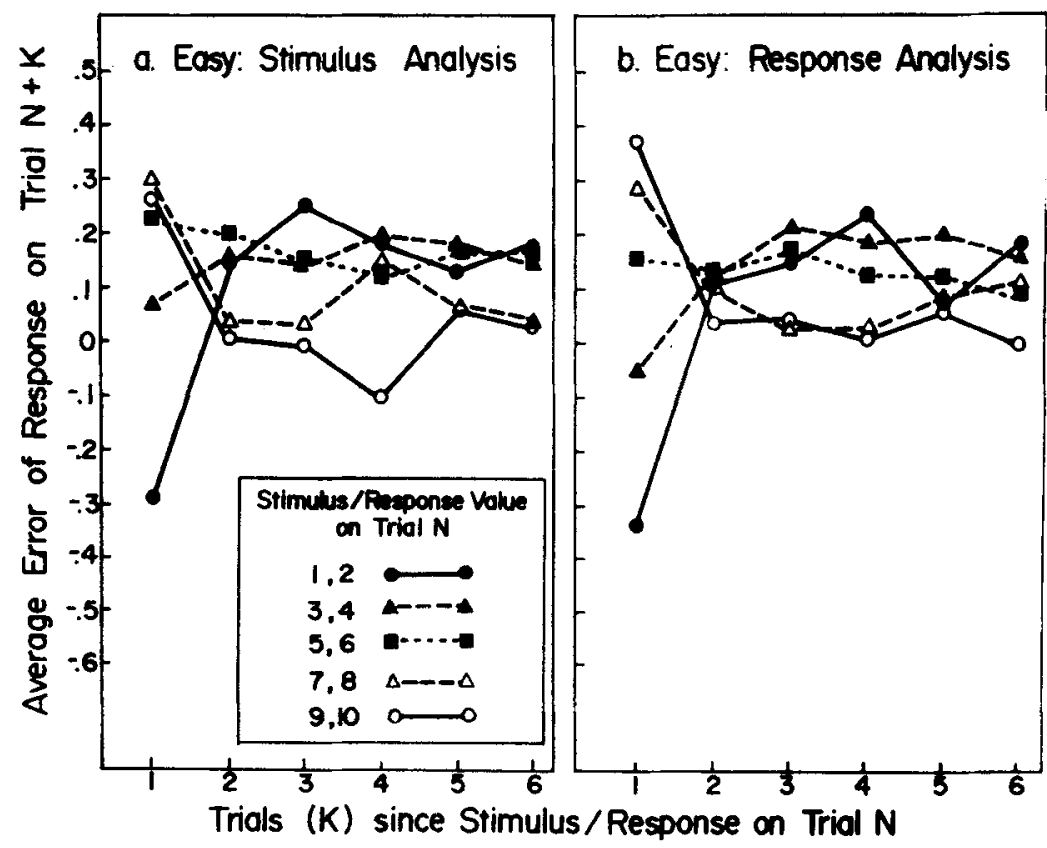

assimilation to the previous stimulus and response, followed by contrast to stimuli and responses further back in the sequence, in an absolute judgment with feedback study, replicates in a modality other than loudness.

The sequential effects in the difficult condition (Fig. 4) are different from those obtained when the stimuli are relatively easy to judge (Figs. 1 and 3), al though the Ss' task was the same in all three conditions. The first-order assimilative effect is smaller in the response analysis than in the stimulus analysis, a result that had not been observed earlier. Apparently the performance manipulation (Fig. 3 vs Fig. 4) affects the first-order sequential effects without markedly changing the higher-order effects, while the feedback manipulation (Fig. 1 vs Fig. 2) affects both the first-order and higher-order sequential effects. It is particularly noted that there is a large amount of assimilation to the previous response and much less assimilation to the previous stimulus when feedback is not provided, while just the reverse is true when the task is difficult but feedback is provided.

\section{STUDY 3: SEQUENTIAL EFFECTS WITH NO STIMULUS PRESENTED}

In this study we asked Ss to perform in an "absolute judgment without stimulus" experiment with feedback provided. The purpose of the study was to determine the

Fig. 4. Average error of the response at Trial $\mathbf{N}+\mathbf{K}$ attributable to the stimulus/response at $\mathrm{Trial} \mathbf{N}$ for the difficult line-length study. (There are approximately 1,200 observations per point.) Results
Fig. 3. Average error of the response at Trial $\mathbf{N}+\mathrm{K}$ attributable to the stimulus/response at Trial $\mathbf{N}$ for the easy line-length study. (There are approximately 1,100 observations per point.)

Figure 5 shows the results of the analyses of the sequential effects. Average error means the difference between the mean guess following a particular previous "stimulus" (or "feedback" number) and the average "stimulus" that actually occurred in the trials following that previous "stimulus" or response. The difference between this procedure and the use of 5.5 as the ideal value of the average stimulus following any previous stimulus or response value should be negligible, since the sequence was random and the number of trials considered at each point was large.

There is marked assimilation of the responses (guesses) to the value of the previous stimulus (numeral presented) and no consistent relation between responses on the current and previous trial. The higher-order effects, as in the above feedback conditions, generally showed contrast for both analyses. The remarkable similarity between Fig. 4 (difficult task, feedback given) and Fig. 5 (guessing, feedback given) is strong support for the argument that the two situations are quite similar with respect to the causes of sequential effects. In the limiting case of chance responses, the two conditions may be equivalent.

\section{DISCUSSION}

The particular response $S$ selects for naming a given stimulus in an absolute

The average percent correct was 9.9. judgment situation is determined

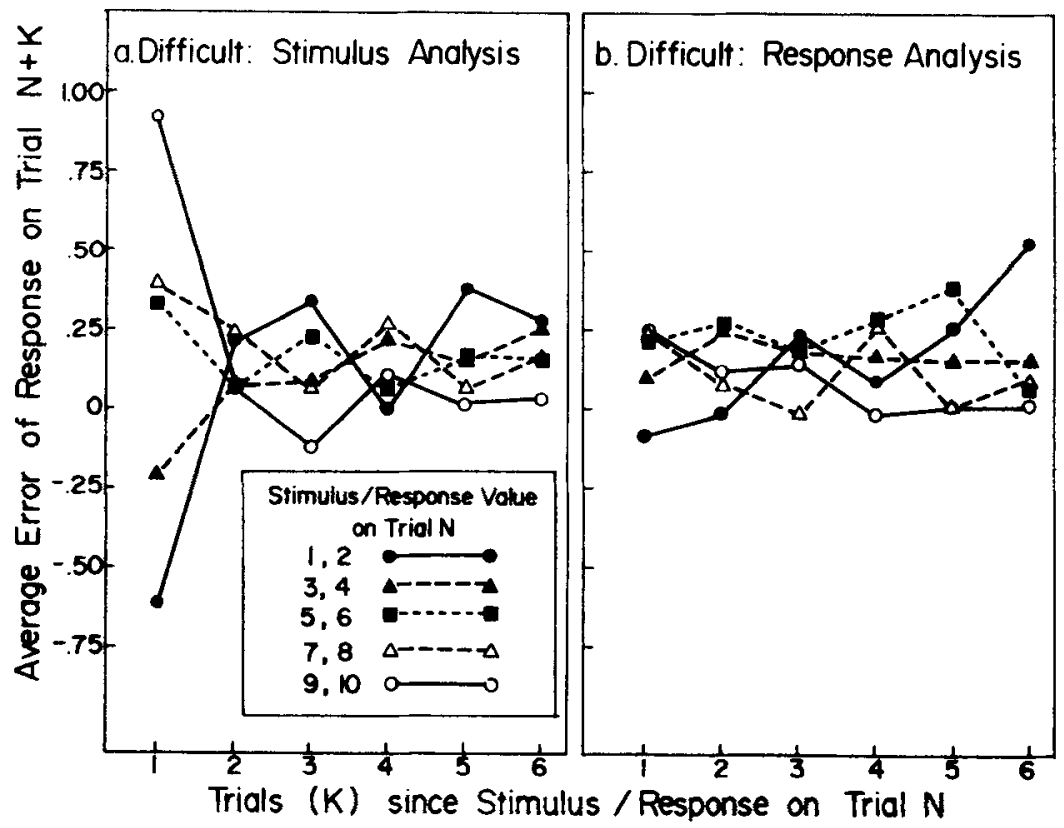




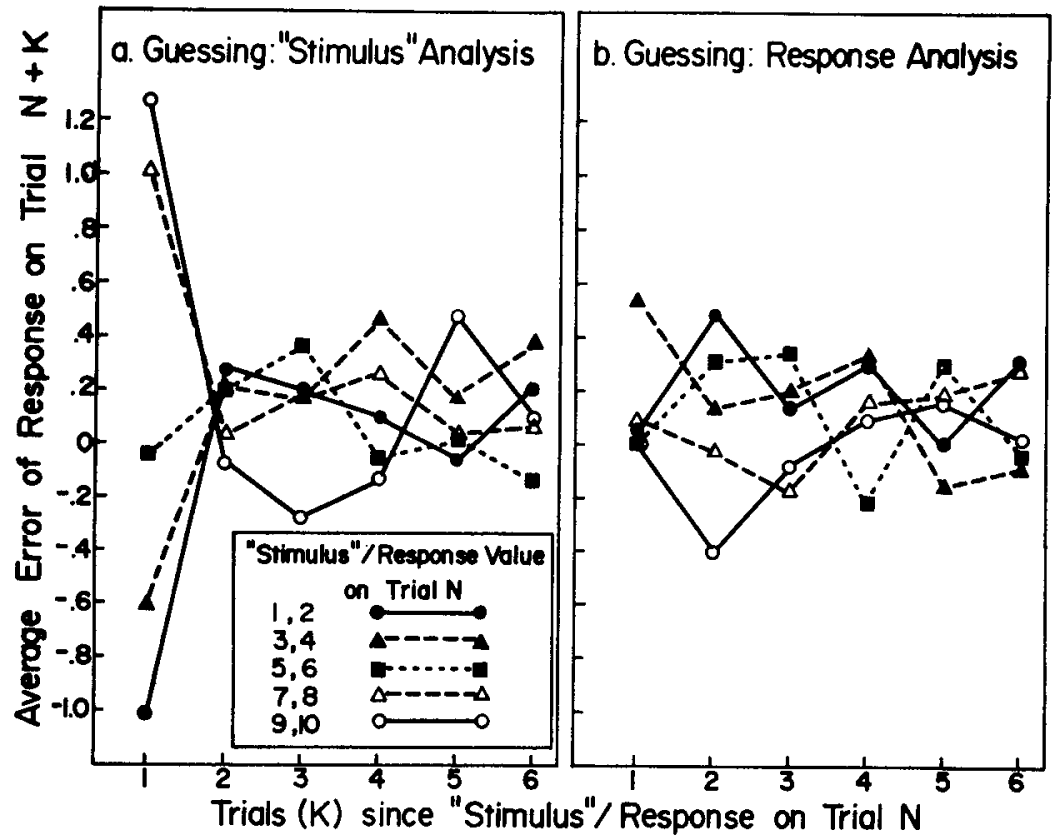

by at least two classes of factors: memory and response or number usage variables. With few stimuli and a relatively large physical spacing between stimuli, identification performance is perfect and, thus, there is no evidence of response processes affecting the judgments. This performance is attributed to memory. As the psychological separation between stimuli is decreased and/or the number of stimuli is increased, Ss begin to make errors, and sequential effects have been observed in all such situations investigated. The reasons for errors are attributed to difficulties of discriminability and to a variable memory or variable criteria. The sequential effects observed when errors are made are attributed to the action of response-system processes which Ss employ when there is uncertainty about the correct response. These two factors are discussed separately below.

\section{The Role of Memory in Judgment}

Long-term memory has been shown to be a factor in absolute judgments. Ward and Lockhead (1970) showed that if the entire stimulus scale is shifted up or down $5 \mathrm{~dB}$ from the level of the previous day, whether or not feedback is provided, the average error of response shifts in the direction of the scale shift. For this to occur, a tendency must exist to respond to the new set of stimuli on the basis of the previous day's scale. The magnitude and form of the sequential effects in that study did not depend on the stimulus scale but were superimposed on the constant errors due to the long-term memory. Thus, it seems that the causes of sequential effects and of long-term memory effects are not related.
In their classic paper on absolute judgment, Wever and Zener (1928) reported that it takes only a few trials for Ss to learn roughly the scale upon which they are to respond in a given experiment. Along with many other investigators, we have also noticed this and 'modify the observation only by small errors due to the long-term memory for previously learned scales. It appears that Ss learn the range over which the stimuli vary in a given situation in a few trials if the range is fixed or if it varies systematically. Thus, after a number of trials there could exist a long-term memory of the part of the total possible range of stimuli being used in the particular study. Ss are thus proposed to have a long-term memory of what the various parts of this range "sound," "feel," "look," etc., like, and of which numbers are to be attached to the various parts.

The judgmental process, then, is viewed as follows. The $S$ compares his "perception" or "short-term memory" of the currently presented stimulus with his long-term memory of the range of stimuli in use. He determines an area of that scale that matches the stimulus. The number assigned to the matching area could then be the response to a particular stimulus. With many stimuli, or a small spacing between stimuli, or a low $S / N$ ratio, the process provides only a rough judgment of the area of the subrange in which the presented stimulus lies. Because of this imprecision of nonverbal long-term memory, the presented stimulus may "match" a large part of the total possible range of stimuli, and $S$ could assign any of several different numbers to the stimulus and still be consistent with all of his stimulus information. Sequential effects
Fig. 5. Average error of the response at Trial $\mathbf{N}+\mathrm{K}$ attributable to the stimulus/response at Trial $\mathbf{N}$ for the guessing study. (There are approximately 600 observations per point.)

are proposed to reflect the S's decision or response processes in determining which of these several possible responses to select.

\section{Response System Processes in Judgment}

We first summarize the pattern of the sequential effects observed. The basic finding is that in most absolute judgment situations the present response is closer to the value of the immediately previous stimulus or response, and is further from the values of stimuli or responses further back in the sequence, than would be expected by chance. For brevity we say there is assimilation followed by contrast but do not imply any relation between these effects and contrast and assimilation as investigated in such areas as simultaneous brightness contrast.

There are two exceptions to the generality. First, when no information feedback is given, assimilation extends to stimuli two trials back and to responses as far as five trials back in the sequence. The magnitude of the assimilation to the previous responses in this case is very much larger than that to previous stimuli.

Second, with feedback given, as performance deteriorates the magnitude of assimilation to the previous stimulus grows larger, while that to the previous response becomes much smaller. When there are no stimuli at all, i.e., when the state of uncertainty as to which is the "correct" response extends to all of the possible responses in the guessing situation, assimilation to the value of the previous "stimulus" is largest of all, and there is no consistent effect of the previous response on the present response.

The effects of stimuli and responses further than one trial back in the sequence generally remain stable and alike as performance decreases to chance in the feedback conditions. But in the no-feedback situation these effects change and it appears that the contrast effects are being canceled by the larger assimilation effects.

Garner (1953) reported data showing that the immediately previous response and stimulus affect the current response in an absolute judgment without feedback situation and that the magnitude of the effect increases as the number of categories (stimuli) increases. McGill (1957), also in a no-feedback study, showed that the contingency between the previous and current responses increases with decreasing 


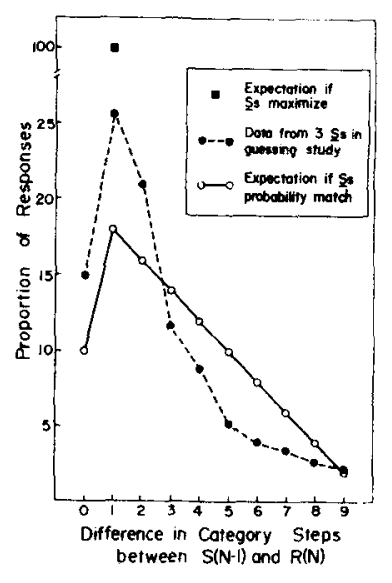

Fig. 6. The frequency distribution of absolute differences between the stimulus at Trial $\mathrm{N}-1$ and the response at Trial $\mathrm{N}$ for the data of the guessing study and the expected frequency distributions if $\mathrm{Ss}$ maximized or probability matched. (There were approximately 3,000 responses available for analysis.)

stimulus discriminability. He reported the contingency in terms of an uncertainty analysis that does not allow determination of its form, and we assume that his result was one of increasing assimilation to the previous response as the task became more difficult. Thus, without feedback assimilation between the previous and present responses increases with task difficulty, while with feedback this increasing assimilation is associated with the previous stimulus (our Studies 2 and 3).

Two guessing strategies are proposed here as possible explanations of these various sequential effects. One of these would account for assimilation and the other for contrast.

The strategy associated with assimilation may be as follows. Consider the probability distribution of possible differences, in category steps, between successive stimuli. When stimuli are presented randomly, this distribution is skewed with a maximum at a difference of one category step. With 10 stimuli presented randomly and equally often, the probability that successive stimuli do not differ (stimulus repetition) is .10 ; that they differ by one step is .18 ; by two steps, .16; and by three through nine steps, .14, .12,.10,.08, .06, .04, and .02 , respectively.

Figure 6 shows the frequency distribution of the differences between the response on Trial $N[R(N)]$ and the stimulus on Trial N - $1[S(N-1)]$ for the three Ss in the guessing study. Also shown are the expected distributions of these differences if Ss followed a strategy of maximization with respect to the probability distribution of differences between successive stimuli and if they probability matched with respect to that distribution. As shown in Fig. 6, Ss guess short steps $(0,1,2)$ more often, and larger steps less often, than they would if they were probability matching. They do not maximize (minimize absolute error). The strategy they appear to adopt is between these extremes. A strategy of guessing mostly small distances necessarily results in "assimilation."

The data from Studies 1 and 2 take the same form as those from the guessing study in Fig. 6. In general, the smaller the magnitude of assimilation, the smaller the deviations from the probability matching curve. We propose that Ss use strategies similar to this guessing behavior when there is a stimulus to be judged but performance is not perfect. After any given stimulus presentation, Ss are likely to be in a state of uncertainty as to which of several acceptable responses is most likely to be "correct." Ss may mediate this uncertainty by choosing, from this subset of acceptable responses, responses that tend to minimize the difference between the value of the previous stimulus and that of the current response. If the "correct" response was on the average in the middle of the group of acceptable responses, the result of this strategy would be assimilation of the current responses toward the value of the previous stimulus (and through the correlation between them to the previous response) regardless of the value of the previous or present stimulus.

For example, consider all those occasions on which an $\mathrm{S}$ is presented with Stimulus 4. Assume that his rough match to his "stimulus scale" always turns up the numbers 3, 4, and 5 as acceptable responses to this stimulus. We would expect that, in the instances where the previous stimulus value was lower than 4 , the $S$ would respond with 3 most often, 4 next most, and 5 least often. Just the opposite would happen in those instances where the previous stimulus was higher than 4 . This means that the average error of response to Stimulus 4 would be negative when 4 was preceded by a lower stimulus and positive when preceded by a higher stimulus; i.e., responses to 4 would be "assimilated" toward the value of the previous stimulus.

In the no-feedback condition $S$ is not given veridical information concerning the value of the previous stimulus. In this case, we assume that $S$ uses the best information available, his own previous response. $\mathrm{He}$ tends toward maximization of response-response differences in the same way as outlined for stimulus-response differences. This would account for the assimilation effect being larger to the previous response than to the previous stimulus in this study, stimuli and responses not being perfectly correlated. Further, if $S$ assimilates his response to the value of the previous response, a compounding of the assimilation effect would be expected, since the previous response itself was assimilated to the response previous to it, and so forth. This would explain why the assimilation effects last so many trials back in the sequence. According to this explanation, the assimilation effect would of necessity grow smaller with increases in the number of trials from Trial $\mathrm{N}$. The more intervening trials, the more different directions of response are interpolated between them, and thus, the more these separate assimilation effects would tend to cancel each other.

The model proposed here is consistent with the fact that the magnitude of assimilation grows larger as performance deteriorates. In a difficult task the presented stimulus carries less information and thus the number of acceptable responses on any particular trial increases. The magnitude of the assimilation effect should increase with any increase in the number of acceptable responses, since tending to minimize the difference between the response and the previous stimulus would result in Ss giving a response that is further away from the "correct" one. This is consistent with a very large assimilation effect in a pure guessing situation.

The contrast of responses to stimuli further back in the sequence might be accounted for in the following way. We know that $S$ tends to use his available responses equally often over a large number of trials (Parducci, 1965). This empirical result may be the average of attempts to use response areas equally often over smaller numbers of trials, perhaps as few as 5 to 10 . We propose that, when presented with a short sequence of stimuli in which the use of one area of the response scale predominates, $S$ adjusts his response scale so as to increase the probability of use of another part of it. This is equivalent to packing category boundaries more closely into areas of previously higher stimulus probability. Thus, for example, following an intermixed serics of low and intermediate responses, 
an intermediate stimulus would tend to be overestimated. This would result in the contrasting of the response to a stimulus occurring after such adjustment with the stimuli occurring before it. The invariability of these effects over performance indicates that this tendency operates regardless of the information carried by the stimulus and, thus, is a fairly stable response-system process. That contrast does not appear until more than two trials back in the no-feedback study is attributed to the compounding of the assimilative error canceling out the smaller contrast effects.

\section{Central Tendency or Assimilation}

There are many studies that demonstrate that, in absolute judgment-like tasks, small stimuli are overestimated, large stimuli are underestimated, and intermediate stimuli generally have no systematic error associated with them. This observation, first made by Hollingworth, has been called the "central tendency effect" and has been attributed to a statistical effect due to imperfect discrimination (Guilford, 1954). Johnson and Mullally (1969, p. 206) state that "when the category judgment is treated as a matching task, correlation analysis leads to a simple explanation of central tendency."

However, if assimilation to a previous stimulus is a fact, and if stimuli are distributed with equiprobability over the range, then: $P\left(S_{N-1}>S_{N}\right)>$ $\mathrm{P}\left(\mathrm{S}_{\mathrm{N}-1} \leqslant \mathrm{~S}_{\mathrm{N}}\right)$ if $\mathrm{S}_{\mathrm{N}}$ is low, $\mathrm{P}\left(\mathrm{S}_{\mathrm{N}-1}>\mathrm{S}_{\mathrm{N}}\right)$ $\approx \mathrm{P}\left(\mathrm{S}_{\mathrm{N}-1} \leqslant \mathrm{~S}_{\mathrm{N}}\right)$ if $\mathrm{S}_{\mathrm{N}}$ is medium, $\mathrm{P}\left(\mathrm{S}_{\mathrm{N}-1}>\mathrm{S}_{\mathrm{N}}\right)<\mathrm{P}\left(\mathrm{S}_{\mathrm{N}-1} \leqslant \mathrm{~S}_{\mathrm{N}}\right)$ if $\mathrm{S}_{\mathrm{N}}$ is high, where $P\left(S_{N-1}>S_{N}\right)$ is the probability that the stimulus value on Trial N-1 is greater than the stimulus value on Trial $\mathrm{N}$. If assimilative effects are constant over stimuli, then the effect is proportional to the probabilities above, i.e., average error is positive if $S_{N}$ is low, average error is zero if $S_{N}$ is medium, average error is negative if $S_{N}$ is high. That is, if $S_{N}$ is low, the probability that the previous stimulus was larger than $S_{N}$ is great, and, thus, if $R_{N}$ is assimilated to the value of the previous stimulus, there will result a positive average error.

On the above level of analysis, central tendency and assimilation are equivalent. The two points of view lead to divergent predictions, however, with a more molecular analysis of the data. Consider, for example, the situation when a low- or a high-valued stimulus is presented in an absolute judgment situation and is followed by an intermediate stimulus. The central tendency or correlation point of view predicts that errors in the average response to the intermediate stimulus would be in the direction of the mean of the stimulus distribution. The assimilation point of view predicts that the intermediate stimulus would be underestimated when preceded by a low-valued stimulus and overestimated when preceded by a high-valued stimulus.

The data presented in both Holland and Lockhead (1968) and Ward and Lockhead (1970) support the assimilation interpretation. For example, in Fig. 1 in Ward and Lockhead (1970), the average error of responses when Stimulus 3,4 is preceded by 1,2 is -.25 , but when the same stimulus is preceded by 9,10 , the average error is +.60 . There is some variability in the data, but this result obtains in general. Responses are assimilated toward the previous stimulus rather than toward the mean of the stimulus distribution. This analysis breaks down at the extremes (Stimuli 1,2 and
$9,10)$ because errors can be in only one direction; i.e., since Stimulus 1 cannot be underestimated and 10 cannot be overestimated because of the limits on the response scale, any occasional large errors in judging these stimuli must produce mean errors that are toward the mean of the distribution. This leads to an apparent central tendency effect for these cases only. However, it still appears more reasonable to attribute these average response effects to an assimilation process than to a "central tendency effect" such as statistical regression.

\section{REFERENCES}

GARNER, W. R. An informational analysis of absolute judgments of loudness. Journal of Experimental Psychology, 1953, 46, 373-380.

GUILFORD, J. P. Psychometric methods New York: McGraw-Hill, 1954

HOLLAND, M. K., \& LOCKHEAD, G. R. Sequential effects in absolute judgments of loudness. Perception \& Psychophysics, 1968, 3, 409-414.

JOHNSON, D. M., \& MULLALLY, C. R Correlation-and-regression model for category judgments. Psychological Review, 1969, 76, 205-215.

LOCKHEAD, G. R. Effects of dimensional redundancy on visual discrimination. Journal of Experimental Psychology, 1966, 72, 95-104.

McGILL, W. J. Serial effects in auditory threshold judgments. Journal of Experimental Psychology, 1957, 53, 297-303.

PARDUCCI, A. Category judgment: A range-frequency model. Psychological Review, $1965,72,407-418$.

WARD, L. M., \& LOCKHEAD, G. R. Sequential effects and memory in category judgments. Journal of Experimental Psychology, 1970, 84, 27-34.

WEVER, E. G., \& ZENER, K. E. The method of absolute judgment in psychophysics. Psychological Review, 1928, 35, 466-493.

(Accepted for publication May 15, 1970.) 